\title{
Implementing Archimedean Spiral Approach to Evaluate Left Ventricular Myocardial Functions
}

\author{
Yashbir Singh ${ }^{1}$, Deepa ${ }^{1}$, Shi Yi Wu ${ }^{1}$, Michael Friebe ${ }^{2}$, Joao Manuel R. S. Tavares ${ }^{3}$, Hu Wei-Chih ${ }^{1}$ \\ ${ }^{1}$ Department of Biomedical Engineering, Chung Yuan Christian University, Zhongli, Taiwan \\ ${ }^{2}$ Electrical Engineering and Information Technologies, Otto-von-Guericke-University, Magdeburg, \\ Germany \\ ${ }^{3}$ Instituto de Ciência e Inovação em Engenharia Mecânicae Engenharia Industrial, Departamento de \\ Engenharia Mecânica, Faculdade de Engenharia, Universidade do Porto, Porto, PORTUGAL \\ weichihhu@gmail.com; weichihhu@cycu.edu.tw; Tel: +886920800097
}

\begin{abstract}
Heart disease can be determined by the calculating regional and global wall motion of the left ventricular (LV). In this research, we designed a dynamic simulation tool using Computed Tomography (CT) images that helps to find the difference between actual and simulated left ventricular functions. In this study, thirteen healthy subjects were involved with actual and simulated left ventricular functions. We obtained the high correlation between actual left ventricular wall motion (ALVWM) and simulated left ventricular wall motion (SLVWM) which is $(r=0.99)$. Our results validate that our simulation tool is feasible for simulating left ventricular motion.

Keywords: Left-Ventricular remodeling, left ventricular wall motion, Archimedean spiral, Myocardial functions, Computer Tomography.

\section{Introduction}

Cardiovascular disease has recognized as the leading causes of death in the entire world. WHO report suggest that 17.7 million people died from CVDs in 2015, representing $31 \%$ of all global deaths. Approx 7.4 million were died due to coronary heart disease and 6.7 million were due to stroke. In 2013 there were $>54$ million deaths globally because of CVDs. The majority of CVD deaths were attributable to either ischemic heart disease (IHD). Over the last few decades, chronic diseases such as cardiovascular disease (CVD), have accounted for a significant fraction of death tolls worldwide. It is well known that smoking, obesity, blood pressure and diabetes mellitus are major risk factors for CVD [1]. In addition, it has also been recognized that many CVD risk factors are associated with each other. It is also seen that heart wall motion can be an indicator for predicting cardiovascular disease in groups of patients with myocardial infarction (MI), unstable angina, typical chest pain, and congestive heart failure (CHF) [2]. left ventricular contractile abnormalities can be an important manifestation of coronary artery disease. These wall motion changes may represent ischemia or infarction of myocardium [3]. This study has been performed to calculate wall motion between actual and simulated LV wall motion and to show correlation study between endocardial displacements to the central axis.
\end{abstract}




\section{Methods}

The objective of this research was to simulate LV functions and to access local left ventricular myocardial function.

In house developed program was integrated with OpenGL library as a tool for analyzing 4D cardio CT image data set. Images were obtained from the computerized tomography (CT) SIEMENS_LEOVB30B instrument provided by Taipei Veterans General Hospital. This study and the informed consent procedure were approved by the Institutional Review Board of Taipei Veterans General Hospital. In this research, thirteen subjects were involved with atrial fibrillation and left ventricular motion abnormalities. Each subject has 10 sets of timing frames, including a complete heartbeat cycle. The scanned image size was $512 \times 512$ (pixels). The images were read by the program and confirmed the format for the DICOM (Digital Imaging and Communications in Medicine) and stored in 512x512 gray formats. 3D reorganization and image information was obtained to construct the stereoscopic model of the thoracic area. We set the left ventricular central axis and image was resampled. The left ventricle region was segmentated to enhance the Eigen value of the left ventricular edge. After that, each layer of the image was multiplied by the resample length, breadth resolution and actual layer of thickness. Endometrium was divided into 31 layers and each layer again divided into 930 points in which 30 points have been taken for our study considering $31^{\text {st }}$ layer as the reference layer. After setting the angle and height, we obtained actual left ventricular information at time 0 and calculated the rate of radius change to simulate left ventricular motion. We performed the analysis of the total volume and RVC (rate of volume change) $[4,5]$.

i) We used manually cutting of LV images from base to apical from the entire data set. One image was selected from the dataset and one seeded point was set to start this process. We obtained the geometrical center that connects each slice on long axis, applied regression calculation to find the line as a reference axis for LV (Fig.1) [6] .
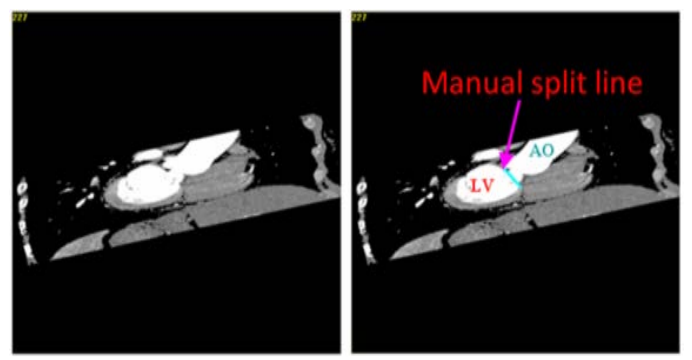

Figure 1. Manually separated line between left ventricle and the aorta

ii) The left ventricular endocardial circle was used for the regional growth of the image which located near to the edge of the image. The regional growth was required to set up the initial seed point. The initial point of the first slice was considered as the reference point of the second slice, the second reference point was set as the seeded region for other layers simultaneously (Fig 2). It was necessary to confirm that the seeded spots of each layer are in the endometrial region and if not, we need to manually correct the seed points $[7,8]$. 


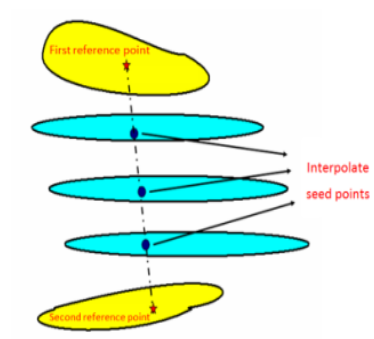

Figure 2. Seeded point star from the first layer and the last layer

iii) In the simulation of left ventricular motion, we got ten sequence of the LV segment from mitral valve to the apical part which represent the distance of the long axis of the left ventricle. Left ventricle contracts with the long axis shorten and the myocardium twist towards the leftward direction then LV wall exhibit thick. The left ventricular end diastolic radius rates of change were calculated. The rate of change in radius and torsion angle were linearly divided into each degree of torsion then the LV contraction model developed shorten axis LV model and radius change torsion model.

iv) Left ventricular systolic contraction describes a uniform movement and relates to the Archimedean spiral equation. Archimedes spiral equation is mentioned below, each $\theta$ value has a corresponding $r$, and the different values of $\theta$ correspond to different $r$ values ( $\cot \alpha \neq 0$ ). If $\cot \alpha>0$, the curve converges would near the pole when $\theta$ approaches to $\infty$, if $\cot \alpha<0, \theta$ approaches $-\infty$, when applying the left ventricular simulated motion, the larger the angle of contraction changes in the larger amount. However, considering the ratio of the change of the inner $r$ value of the Archimedes spiral at 180 degrees is too small then turn the angle of twist by 180 degrees (Fig.3) [9].

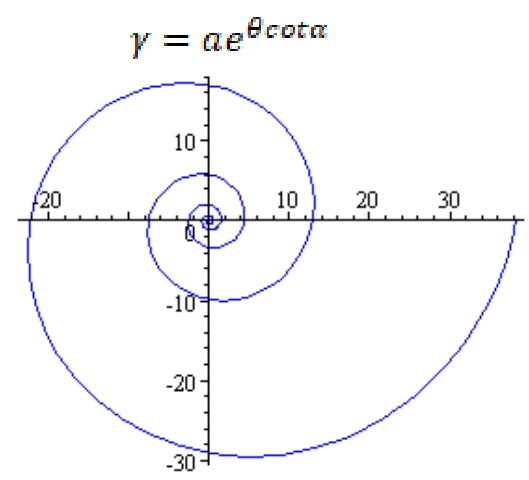

Figure 3. Archimedean Helix

v) The radius rate change was calculated by taking average of the 30 sampling points to the center of each layer and also calculated the actual average final radius of contraction. Whenever a twist occurs, the mean radius of end-diastole and $\mathrm{R}$ rate $=$ (RED radius of end-diastole -RES) / RED because we set the maximum twist angle to 20 degrees as shown in equation (ii). The red is the spiral created by the original Archimedes and the blue is by adding a spiral of rate of change in radius. It shows that the radius of the blue line will vary more widely with the angle (Fig.4) [10] .

$$
R=\gamma \times\left(1+\left(\frac{R_{\text {Yate }}}{20} \times \text { angle }\right)\right)
$$




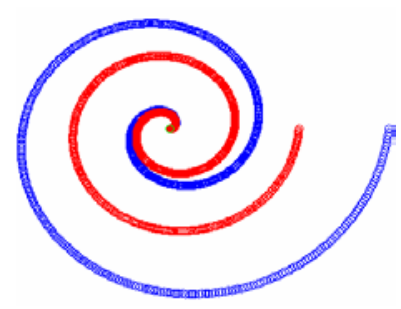

Figure 4. Archimedean spirals plus situational change rates.

Left ventricle before and after the simulation is shown in the figure. A1 represents the right half of the point and $A 2$ represents the left half of the point. The left and right sides of $A 2$ and the left hemisphere makes a distance contraction change in the way to the left, the left ventricle after simulation (A1) and A2 Points change to B1 and B2 respectively (Fig.5).
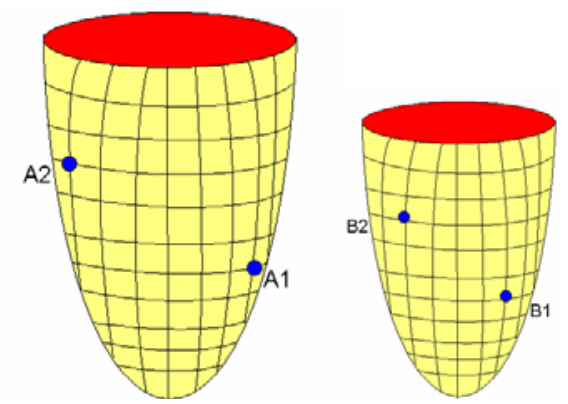

Figure 5. Left ventricle simulation (A1 and A2 before the displacement, Angle and height changes to B1 and B2

\section{Results}

We proposed a novel approach to evaluate the left ventricular myocardial functions and the normalized wall thickness, which can be used to detect wall motion abnormality. This feature considers the variations between normal and abnormal contraction by tracking the normalized thickness of all segments between the endo and epicardium during the whole cardiac cycle. In this work, the simulated data was validated against real data. The volume difference in each time frame and data set was evaluated. Here we have discussed the result in different steps.

\section{Simulation Interface:}

The input parameters shown in the red box. The simulation was performed on 1 degree interval and one-time left-heart grid modeled with 0 to 20 degrees of variation. Each simulated angle is shown in green box (Fig 6) (Fig 7). 
Yashbir Singh, Deepa, Shi Yi Wu, Michael Friebe, Joao Manuel R. S. Tavares, Hu Wei-Chih; Implementing Archimedean Spiral Approach to Evaluate Left Ventricular Myocardial Functions. Journal of Biomedical Engineering and Medical Imaging, Volume 5, No 3, June (2018), pp 1-8

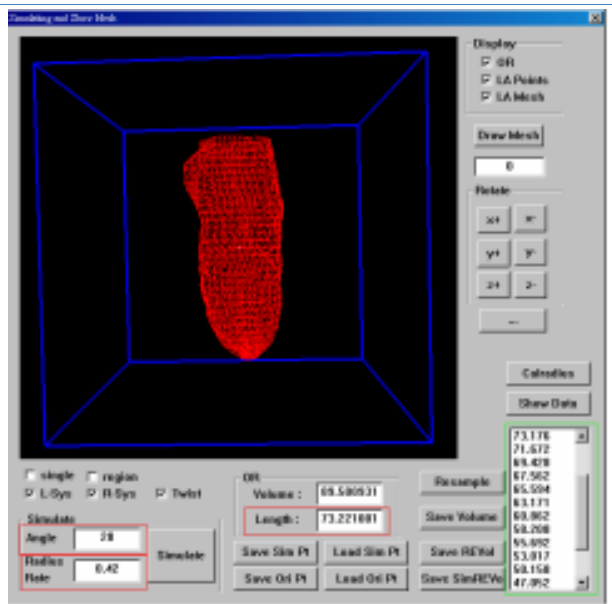

Figure 6. Red box is shown angle and radius rate, Green box is shown long axis parameter. Simulated volume is shown on 0 to 20 degrees

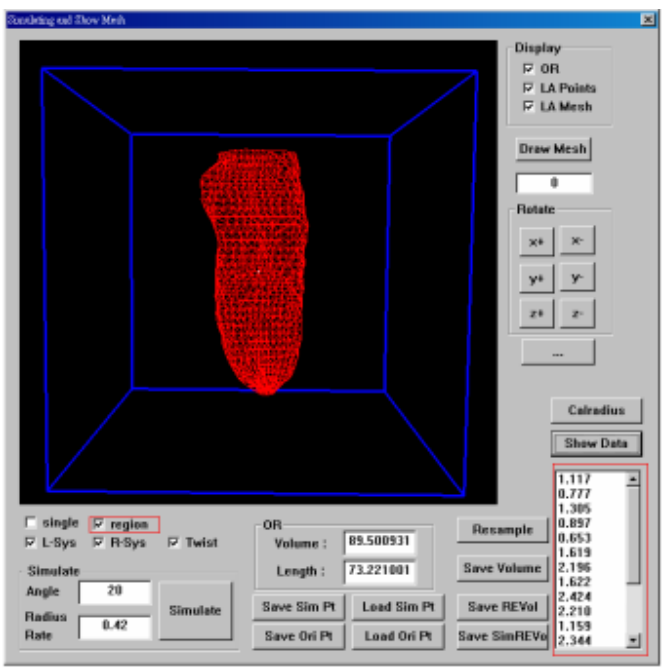

Figure 7. Red box is shown the volume of the local 18 regions.

\section{Parameter Analysis Display Interface}

Comparison of the left ventricular function is shown. The red line represents actual VTC and RVC while the green line represents the simulated value. This observation window clearly shows that similarity between the constructed simulation system and the real left ventricle. This confirms the relevance of our system for global left ventricular function between simulated and real left ventricular changes in motion (Fig.8) . 


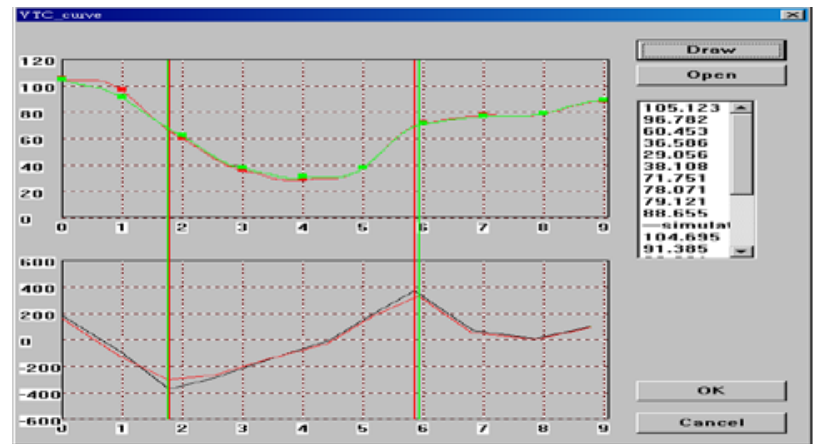

Figure 8. RVC and VTC display interface (red line) as real data (green line) as analog data

\section{Dynamic and Analysis Display Interface}

The dynamic interface allows observing more clearly actual and simulated left ventricular motion.

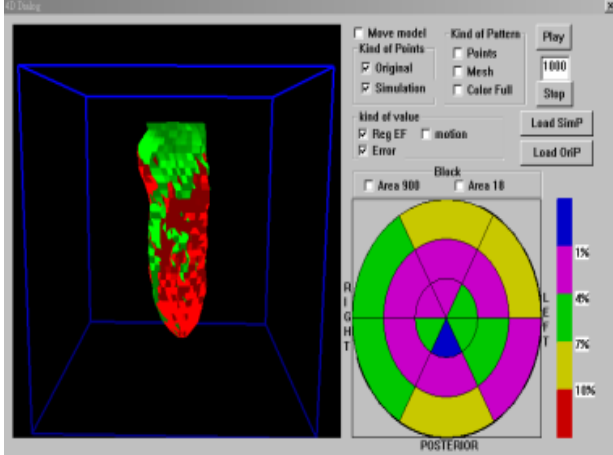

(A)
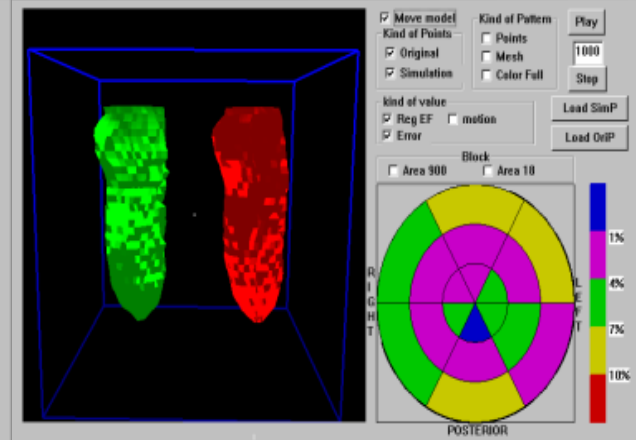

(B)

Figure 9 Dynamic Interface (A) Actual vs. Simulated Left Ventricle (B) Separated Actual vs Simulated Left Ventricle

In the comparative study of actual and simulated heart, we got the variation on longitudinal axis which is $1 \%$ (purple), $4 \%$ (green), $7 \%$ (green) and $10 \%$ (red) on various points of heart wall (Fig.9).

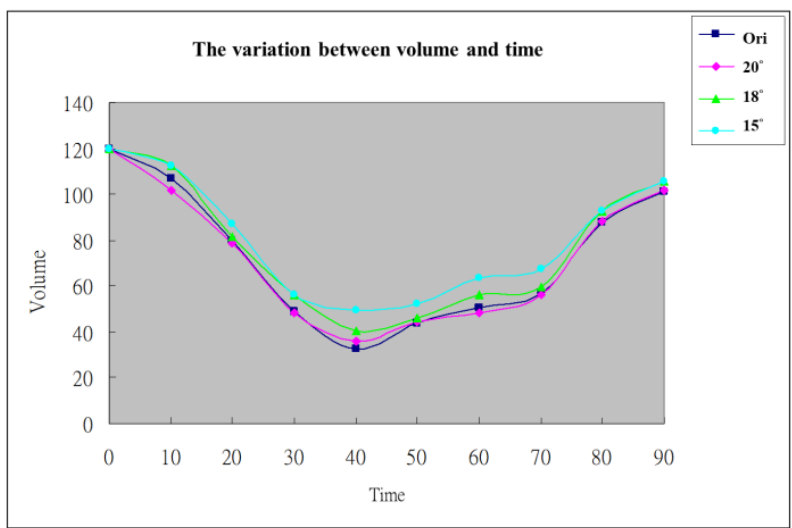

Figure 10. Angle and volume changes in the relationship diagram

This is an initial step to recognize local and global dysfunction in the heart. This study tested the effect of torsion angles which were set at $15^{\circ}$ (cyan), $18^{\circ}$ (green), and $20^{\circ}$ (purple) (Fig.10). The magnitude of left ventricular volume change is relatively smaller when the angle of the input is smaller as well. The calculation of left ventricular function may cause misjudgment. Therefore, we set the torsion angle to $20^{\circ}$ in order to meet the change of real left ventricular volume (Table1,Table 2). 
Yashbir Singh, Deepa, Shi Yi Wu, Michael Friebe, Joao Manuel R. S. Tavares, Hu Wei-Chih; Implementing Archimedean Spiral Approach to Evaluate Left Ventricular Myocardial Functions. Journal of Biomedical Engineering and Medical Imaging, Volume 5, No 3, June (2018), pp 1-8

Table 1 The radius change of 13 subjects

\begin{tabular}{|c|c|c|c|}
\hline Subject & $\begin{array}{c}\text { Radius rate of } \\
\text { change }\end{array}$ & Subject & $\begin{array}{c}\text { Radius rate of } \\
\text { change }\end{array}$ \\
\hline Subject1 & 0.48 & Subject8 & 0.41 \\
\hline Subject2 & 0.46 & Subject9 & 0.33 \\
\hline Subject3 & 0.30 & Subject10 & 0.44 \\
\hline Subject4 & 0.42 & Subject11 & 0.43 \\
\hline Subject5 & 0.32 & Subject12 & 0.39 \\
\hline Subject6 & 0.45 & Subject13 & 0.37 \\
\hline Subject7 & 0.47 & & \\
\hline
\end{tabular}

Table 2. left ventricular volume differences

\begin{tabular}{|c|c|c|c|}
\hline Time & Original $(\mathrm{ml})$ & Resample $(\mathrm{ml})$ & Difference $(\mathrm{ml})$ \\
\hline 00 & 134.5 & 132.4 & 2.1 \\
\hline 10 & 114.1 & 112.3 & 1.8 \\
\hline 20 & 77.3 & 76.1 & 1.2 \\
\hline 30 & 46.7 & 45.9 & 1.2 \\
\hline 40 & 40.7 & 40.1 & 0.6 \\
\hline 50 & 56 & 55.1 & 0.9 \\
\hline 60 & 84.1 & 82.8 & 1.3 \\
\hline 70 & 96.1 & 94.6 & 1.5 \\
\hline 80 & 104.1 & 102.5 & 1.6 \\
\hline 90 & 116.8 & 114.9 & 1.9 \\
\hline
\end{tabular}

\section{Conclusion}

In this research, we developed simulation tool using 3D images of actual left ventricular on long-axis changes that tool helps in the accurate assessment of cardiac regional function. We evaluated the difference between the actual and simulated left ventricular function. The difference with the simulated model shows to assess the myocardial position of LV and shown using the myocardial twisting function and shortening of long axis can accurately simulate the LV function. Further research will involve the integration of the heart motion, validating geometrical landmarks and integration of the motion matching.

\section{REFERENCES}

[1] Kones, R. Primary prevention of coronary heart disease: integration of new data, evolving views, revised goals, and role of rosuvastatin in management. A comprehensive survey. Drug design, development and therapy, 2011.

[2] Cicala, Silvana, et al. "Prevalence and prognostic significance of wall-motion abnormalities in adults without clinically recognized cardiovascular disease: the Strong Heart Study." Circulation, 2007. 
[3] Park, J., Metaxas, D., Young, A., \& Axel, L. Model-based analysis of cardiac motion from tagged MRI data. In Computer-Based Medical Systems, 1994., Proceedings 1994 IEEE Seventh Symposium on (pp. 40-45). IEEE. 1994.

[4] Monga and N. Ayache. From voxel to curvature. IEEE Computer Vision and Pattern Recognition, 1991, 644-649.

[5] P. T. Sander and S. W. Zucker. Inferring surface trace and differential structure from 3D images. IEEE Transactions on Pattern Analysis and Machine Intelligence, 1990, 12(9):833-854.

[6] J. Park, D. Metaxas, A. A. Young, and L. Axel, "Deformable models with parameter functions for cardiac motion analysis from tagged MRI data, "IEEE Transactions on Medical Imaging, 1996.

[7] J. Huang, D. Abendschein, V. G. Davila-Roman, and A. A. Amini, "Spatio-temporal tracking of myocardial deformations with a 4-D B-spline model from tagged MRI," IEEE Transactions on Medical Imaging, 1999.

[8] Adams R, Bischof L. Seeded Region Growing. IEEE Transactions on pattern analysis and machine intelligence 1994: 16:641-647.

[9] Walser, H.; Hilton, P.; Pedersen, J.; Mathematical Association of America. Symmetry. Mathematical Association of America. p. 27. ISBN 9780883855324. Retrieved 2014-10-06.

[10] Kass, M., Witkin, A., \& Terzopoulos, D. Snakes: Active contour models. International journal of computer vision, 1(4), 1998. 